\title{
What is the available evidence that artificial light at night affects animal behaviour? A systematic map protocol
}

\author{
Lucy Katherine McLay ${ }^{1 *} \mathbb{D}$, Juhani Peter Hopkins² ${ }^{2}$ Bob Bern Ming Wong ${ }^{3}$, Ulrika Candolin ${ }^{4}$ \\ and Therésa Melanie Jones ${ }^{1}$
}

\begin{abstract}
Background: Artificial light at night (ALAN), has increased dramatically over the past two centuries and is linked to demonstrable shifts in a range of behaviours across diverse animal taxa. This systematic map will collate and synthesise the documented effects of ALAN on animal behaviour and fitness, identify gaps in the literature, inform future research and provide the basis for a decision-making tool for informing policy makers.

Methods: This systematic map will summarise and examine all available evidence on the effects of ALAN on animal behaviour and ensuing fitness effects. All documented changes to behaviour in animals (excluding humans), will be included and both peer reviewed primary and grey literature will be searched. Searches will be conducted in academic journal databases, online search engines, and specialist websites. Articles will be screened for inclusion in the systematic map at title, abstract and full-text levels and will then be critically appraised for study robustness and validity. Data from studies included in the review will then be extracted and coded according to categories informed by consultation with Stakeholders. Data will be summarised, where possible, in a quantitative manner, accompanied with a descriptive overview. Future avenues for research and specific questions suitable for a systematic literature review will be formulated.
\end{abstract}

Keywords: Circadian, Fitness, Lighting design, Light pollution, Migration, Reproduction, Skyglow, Street light, Urban environment

\section{Background}

In the last two centuries there has been a rapid global increase in the extent and intensity of the anthropogenic production of light at night [1]. Widespread light radiation into non-targeted regions of the environment, means that artificial light at night (ALAN) is currently one of the most pervasive, but unrecognised, forms of environmental pollution [1-3]. ALAN is largely concentrated around lit infrastructure in terrestrial urban environments, however, it is also present in many coastal and marine ecosystems due to lit industrial installations [2]. Additionally, light from urban areas may be emitted horizontally or

\footnotetext{
*Correspondence: Imclay@student.unimelb.edu.au

1 School of BioSciences, Faculty of Science, The University of Melbourne, Melbourne, VIC 3010, Australia

Full list of author information is available at the end of the article
}

reflected by cloud cover or airborne particles, causing sky-glow to extend tens to hundreds of kilometres from the urban boundary [4]. Animal species living in such light polluted areas may never experience natural night darkness. Furthermore, ALAN is anticipated to continue to increase, with $60 \%$ of the global population predicted to live in cities by 2030 [5]. Worryingly, many new urban developments are proposed for areas currently in natural darkness at night.

Life on earth evolved under a daily and seasonal cycle of a relatively bright day (up to 200,000 lx) and a dark night (up to $0.03 \mathrm{~lx}$ ) $[6,7]$. Consequently, animal behavioural and physiological traits are synchronized to this daily and seasonal rhythm $[8,9]$. Disruption to the natural photic environment has been linked to changes in a suite of behaviours and physiological mechanisms, reductions in fitness, declines in species abundance and shifts 
in species interactions and community composition [8]. The behavioural effects observed for animals exposed to ALAN are very broad and include alteration to daily activity patterns, juvenile orientation, courtship and mating, migration and changes to seasonal rhythms $[7,8,10$, 11]. They are also taxonomically widespread. The mechanisms underpinning the observed shifts are not well understood and differ depending on the affected trait and across species, but evidence suggests it can involve changes to light mediated activity and migration cues, as well as alteration to light-sensitive hormone production, which drives circadian rhythms and physiological processes [12]. These changes can lead to modifications in species interactions and predator/prey relationships [13].

Investigation of the ecological effect of ALAN on animal behaviour is a burgeoning field [3]. A systematic literature map of the effects of ALAN on animal behaviour is warranted to map the range of behavioural effects across species groups, identify gaps in knowledge and to direct future research. Currently, global lighting strategy policies are driven principally by issues associated with human safety and security and to achieve reductions in energy expenditure and overall costs. By contrast, the biological effects of ALAN on animals has been largely ignored by strategists and policy makers [3].

We consulted with academics working in the field of ALAN and animal behaviour to help formulate the main and secondary question and then define the scope of the systematic map. It was agreed to include behavioural effects of ALAN at night where there was a difference in intensity between day-time and night-time light (excluding effects of constant bright light), but not behavioural impacts on humans. Additional stakeholder engagement to inform the parameters of the literature to be captured, will be undertaken before the map is undertaken with: academics working the field of Urban Planning; industry consultants working in Sustainability Planning; and, Federal, State and Local Government Sustainability Planners.

This systematic map will consolidate the evidence for the importance of ALAN as a pervasive form of environmental pollution which affects behaviour across taxa. It will inform future research into the effects of ALAN, aid decision making of urban lighting strategies and direct conservation initiatives to maintain wildlife stability and diversity in the face of global increases in the intensity and spread of artificial light at night.

\section{Objective of the review}

The objective of this map is to collate, code and configure all relevant evidence on the effects of ALAN on the behaviour of animals, excluding humans. Where possible we will specify identified effects on animal fitness as a secondary question. We aim to ascertain gaps in the literature, possible future research areas and specific literature review questions. The results could be used as the basis for a decision-making tool for urban light planning.

\section{Question \\ Main question}

What is the available evidence that artificial light at night affects animal behaviour?

\section{Secondary question}

Do changes in animal behaviour caused by artificial light at night have any consequences for animal fitness?

\section{Components of the primary question Subject}

Any measurable behaviours in all animals (excluding humans) and fitness consequences (defined as effects on individual lifetime reproductive success and/or survival).

\section{Exposure}

Anthropogenically produced light at night, where there is a diurnal difference in levels of light between day-time and night-time light (i.e. not constant bright light).

\section{Comparator}

Animal behaviour under natural levels of light at night (stars and moon) or dark night control. Studies could encompass before/after study design, different exposure areas and laboratory based experiments.

\section{Outcomes}

Changes to any measurable behaviours and subsequent fitness in animals.

\section{Methods}

\section{Searching for articles \\ Search strategy}

The search aims to collect a comprehensive selection of scientific reports on the effects of ALAN on animal behaviour in an unbiased way. The systematic map follows collaboration for environmental evidence (CEE) guidelines [14] and complies with reporting standards for systematic evidence synthesis (ROSES) [15] (see Additional file 1 for ROSES checklist). To improve the likelihood of capturing a wider range of papers, we will conduct the search using a variety of databases and search engines, with the time of each search documented. All search strings for each search conducted will be provided as additional information in the systematic map.

\section{Search terms}

We have conducted a scoping exercise to test alternative search strings. Search terms for intervention/exposure 
and a wide range of subjects (potential behaviours) were formulated using the artificial light at night (ALAN) research literature database (http://alandb.darksky.org). No terms for animal taxa were included, because scoping indicated that many reports use specific taxonomic information that cannot be captured using animal taxa group names.

The search terms we will use are:

Intervention/exposure Anthropogenic light at night, light at night, light pollution, man-made light, nightlight, nocturnal illumination, part night light, skyglow, skyplot, streetlamp, streetlight, urban light, vertical light beam.

Subject Active, activity, aggregate, anxiety, attract, bask, behaviour, birth, breed, call, choice, chorus, circadian rhythm, collide, communicate, commute, consume, court, courtship, cue, diapause, disperse, drift, eclosion, ecology, emerge, feed, fitness, flight, fly, forage, hatch, kill, learn, mate, migrate, move, navigate, nest, orientate, oviposit, play, pollinate, predation, predator, prey, reproduction, roost, run, sea-finding, signal, sing, sleep, song, stress, swim, vocalise, walk.

As applicable to databases, all search terms will be truncated and written with a wildcard at the end to include alternative forms of the word (e.g. forage $=$ forag* to include foraging, forages, forager). Wildcards will be used where necessary to include alternative spelling and hyphenation of words. When possible, a search will be conducted by combining all individual terms of each category with the Boolean term OR operator and the two categories combined using the Boolean AND operator. Scoping revealed a large number of articles on the unrelated topics of astronomy, cancer research and traffic planning, so we will use these words with the Boolean NOT operator to exclude results when possible. A test search was conducted to ensure that the use of the NOT operator did not exclude any relevant articles. For example, in Web of Science the following search string will be used:

TS = ("light at night"*" OR "anthropogen" light"*" OR "urban light" " OR "light pollution*" OR "nightlight"* OR "man made light"” OR "part night light"” OR "street light" OR streetlight" OR "vertical light beam*" OR "street lamp" OR streetlamp* OR "sky glow"” OR "skyglow")

AND

$\mathrm{TS}=$ (behavio" OR "circadian rhythm" ${ }^{*}$ OR migrat" OR forag" OR oviposit" OR reproduct" OR court" OR activ* OR fitness" OR mat" OR sleep* OR anxiety* OR predat" OR breed* OR navigat* OR feed* OR communicat* OR song* OR sing" OR call* OR pollinat* OR birth" OR eclos* OR emerg* OR commut* OR diapaus* OR dispers* OR consum* OR cue* OR nest* OR ecolog* OR mov* OR stress* OR signal* OR prey* OR bask* OR learn* OR play* OR choice* OR hatch" OR "sea-finding"" OR fly" OR walk* OR swim* OR run* OR flight OR orientat* OR aggreg* OR colli* OR attract* OR roost" OR chorus* OR vocali?"* OR kill")

NOT

$\mathrm{TS}=\left(\right.$ traffic* $^{*} \mathrm{OR}$ vehicle ${ }^{*}$ OR engineer" OR astronom* OR tumor* OR tumour* OR cancer*)

\section{Abbreviated search}

Where a complex search string is not accepted, searches will aim to include at least one term for subject and one for intervention/exposure. All modified search strings will be recorded. No search restrictions beyond the ones outlined under Search Terms will be applied.

\section{Language}

All searches will be conducted using English search terms and due to time and reviewer limitations, only studies published in English will be included in the Systematic Map.

\section{Estimating comprehensiveness of search}

To evaluate the performance of the search strategy, a test list of 30 articles from a recent review on the behavioural effects of ALAN has been compiled (see Additional file 2 for list of benchmark articles) and will be compared with the results of our search. If any articles are found to be missing from our search, the search string will be amended.

\section{Publication databases}

The search will be conducted using the following online databases:

1. Agricola.

2. Biological abstracts.

3. Ecology abstracts.

4. Environmental Sciences and pollution management.

5. PubMed.

6. Scopus.

7. Sustainability science abstracts.

8. Water resources abstracts.

9. Web of Science.

\section{Websites}

An internet search will be conducted using:

10. http://google.com.

11. http://scholar.google.com.

These website searches will be limited to the first 50 results and Word or PDF documents. 


\section{Specialist sources}

Websites of the listed organisations will be searched for relevant literature.

12. International dark skies association.

13. Artificial light at night (ALAN) research literature database.

14. IUCN website.

15. European commission website.

\section{Article screening and study eligibility criteria Screening process}

Collected articles will be evaluated for inclusion at three successive levels. First, they will be judged for relevance based on their title and those deemed outside the scope of the review will be excluded from further evaluation stages. To check consistency, a subset of 100 article titles or $10 \%$ (whichever is greater) will be assessed by two reviewers. Second, articles will be judged for relevance on abstract. The third level will evaluate each article by studying the full text. Any reviewer who is the author of a study will not decide on the inclusion of that study and, in cases of uncertainty, the reviewer will tend towards inclusion for each review level.

A subset of at least $10 \%$ of the articles at the second and third stage will be assessed by at least two reviewers. Cohen's kappa coefficient will be calculated to determine the level of agreement between reviewers and a score $\kappa<0.6$ will indicate inconsistency. In case of inconsistency, discrepancies will be discussed and the inclusion criteria will be clarified or modified.

\section{Eligibility criteria}

For an article to be included it must fulfil the following criteria:

Eligible subject Any measurable form of animal behaviour (excluding humans).

Eligible intervention/exposure Anthropogenically produced light at night (excluding constant bright day and night-time light) that can constitute ALAN to the animal.

Eligible comparator Animal behaviour under natural levels of light at night (stars and moon) or dark night control.

Eligible outcome Any measurable change to animal behaviour compared to a control. Changes to physiology that do not measurably affect behaviour are excluded.

Eligible types of study design Study designs should be field or laboratory studies of before/after ALAN intervention or of paired sites/treatments with/without ALAN.

A list of articles excluded at the full text stage will be provided as additional material in the final review together with each article's reason for exclusion.

\section{Study validity assessment}

Prior to data extraction, all studies that pass the full-text screening stage will undergo a critical appraisal of the internal and external validity of the study on a threepoint scale (low, medium, high) for a number of parameters, which will include, but not be limited to:

- Study design.

- Study setting (quality of comparable ALAN and no ALAN treatments for both field and laboratory studies).

- Temporal extent of study (short-term/long-term).

- Replication.

- Use of a relevant control.

- Statistical analysis.

The overall validity of the study will be determined by the average score across the parameters and those studies with an average "low" score will be excluded from the review and subsequent synthesis. To ensure repeatable estimation of validity, examples of what constitute low/ medium/high ranking for each parameter will be agreed by reviewers prior to extraction. A subset of $10 \%$ of studies will be assessed by two reviewers. Any reviewer who is the author of a study will not decide on the inclusion of that study and any disagreement between the reviewers will be solved through discussion and if necessary consultation with a third reviewer. Any article excluded will be listed in the additional material together with the reason for exclusion.

\section{Data coding strategy}

Data extracted from studies included in the map will be recorded and coded in an MS-Excel spreadsheet (including the date that data was retrieved for each search/ search engine). Data categories will be agreed following consultation with Stakeholders using an iterative process. Extracted information will include but not be limited to:

- Bibliographic information.

- Animal species/group.

- Night-time vs day-time light intensity.

- Number of hours of day vs night light.

- Lighting specifications or source (including brightness or intensity).

- Spectra/light colour.

- Study location.

- Study type-field/laboratory.

- Study design.

- Behaviour affected.

- Effect on behaviour.

- Effect on fitness.

- Sample sizes. 
- Measured outcome means and measures of variation such as standard error, standard deviation and confidence intervals.

The extracted information will be used to assess the range and extent of effects of ALAN on animal behaviour. If relevant data are not easily decipherable, the corresponding author of the article will be contacted to request the information. To ensure that data are extracted in a way that is repeatable and consistent, two reviewers will extract information from a subset of $10 \%$ of articles. Any inconsistencies in the extracted information will be discussed and the extraction methodology refined.

\section{Study mapping and presentation}

In the final systematic map, we will describe the review process and present evidence of categorised data from all included studies. A flow chart illustrating the study eligibility process with the number of papers included in each stage will be presented. We will use recorded categories to summarize results into tables, figures and geographical maps, accompanied by a narrative review. This systematic map will also identify and highlight major knowledge gaps and clusters in the evidence base for behavioural and fitness effects of ALAN, based on recorded categories.

\section{Additional files}

\section{Additional file 1. ROSES checklist.}

Additional file 2. Benchmark articles.

\section{Authors' contributions}

The initial review questions were developed by BBMW and UC. Development of the systematic review protocol was led by LKM and JPH, with input from all authors. All authors contributed to the manuscript write up. All authors read and approved the final manuscript

\section{Author details}

${ }^{1}$ School of BioSciences, Faculty of Science, The University of Melbourne, Melbourne, VIC 3010, Australia. ${ }^{2}$ Department of Ecology and Genetics, University of Oulu, 90014 Oulu, Finland. ${ }^{3}$ School of Biological Sciences, Faculty of Science, Monash University, Clayton, VIC 3168, Australia. ${ }^{4}$ Department of Organismal and Evolutionary Biology, University of Helsinki, 00014 Helsinki, Finland.

\section{Acknowledgements}

Not applicable.

\section{Competing interests}

The authors declare that they have no competing interests.

\section{Availability of data and materials}

Data sharing is not applicable to this article as no datasets were generated or analysed during the current study.

\section{Consent to publish}

Not applicable.
Ethics approval and consent to participate Not applicable.

\section{Funding}

The project is funded by the Academy of Finland (Grant 24302211), the Jenny and Antti Wihuri Foundation, and a Ben Gurion University of the NegevMonash University collaborative grant. Publication costs are funded by the Ben Gurion University of the Negev-Monash University collaborative grant.

\section{About this supplement}

This article has been published as part of Environmental Evidence Volume 8 Supplement 1, 2019: Using animal behavior in conservation management.

The full contents of the supplement are available online at https://environmen talevidencejournal.biomedcentral.com/articles/supplements/volume-8-suppl ement-1.

\section{Publisher's Note}

Springer Nature remains neutral with regard to jurisdictional claims in published maps and institutional affiliations.

Published: 13 June 2019

\section{References}

1. Kyba CCM, Kuester T, Sánchez de Miguel A, Baugh K, Jechow A, Hölker $F$, et al. Artificially lit surface of Earth at night increasing in radiance and extent. Sci Adv. 2017:3:11.

2. Falchi F, Cinzano P, Duriscoe D, Kyba CCM, Elvidge CD, Baugh K, et al. The new world atlas of artificial night sky brightness. Sci Adv. 2016;2(6):25.

3. Davies TW, Smyth T. Why artificial light at night should be a focus for global change research in the 21st century. Glob Change Biol. 2018;24(3):872-82.

4. Falchi F, Cinzano P, Elvidge CD, Keith DM, Haim A. Limiting the impact of light pollution on human health, environment and stellar visibility. J Environ Manage. 2011;92(10):2714-22.

5. United Nations. The World's cities in 2016_data booklet. Uniter nations-department of economic and social affairs. 2016.

6. Longcore T, Rich C. Ecological light pollution. Front Ecol Environ. 2004;2(4):191-8.

7. Gaston KJ, Duffy JP, Gaston S, Bennie J, Davies TW. Human alteration of natural light cycles: causes and ecological consequences. Oecologia. 2014;176(4):917-31.

8. Navara KJ, Nelson RJ. The dark side of light at night: physiological, epidemiological, and ecological consequences. J Pineal Res. 2007;43(3):215-24.

9. Gaston KJ, Davies TW, Nedelec SL, Holt LA. Impacts of artificial light at night on biological timings. Annu Rev Ecol Evol Syst. 2017;48(1):49-68.

10. McLay LK, Green MP, Jones TM. Chronic exposure to dim artificial light at night decreases fecundity and adult survival in Drosophila melanogaster. J Insect Physiol. 2017;100:15-20.

11. Tierney SM, Friedrich M, Humphreys WF, Jones TM, Warrant EJ, Wcislo WT. Consequences of evolutionary transitions in changing photic environments. Aust Entomol. 2017;56(1):23-46.

12. Reiter RJ, Tan DX, Fuentes-Broto L. Melatonin: a multitasking molecule. Neuroendocrinology. 2010;181:127-51.

13. Bennie J, Davies TW, Cruse D, Inger R, Gaston KJ. Cascading effects of artificial light at night: resource-mediated control of herbivores in a grassland ecosystem. Philosoph Trans R Soc B. 2015;370(1667):40131.

14. Pullin AS, Frampton GK, Livoreil B, Petrokofsky G, Edelstein K, (Eds). Collaboration for Environmental Evidence. 2018. Guidelines and standards for evidence synthesis in environmental management. Version 5.0 2018. http://www.environmentalevidence.org/information-for-authors. Accessed 17 Dec 2018.

15. Haddaway NR, Macura B, Whaley P, Pullin AS. ROSES RepOrting standards for systematic evidence syntheses: pro forma, flow-diagram and descriptive summary of the plan and conduct of environmental systematic reviews and systematic maps. Environ Evid. 2018;7(1):7. 